Molecules 2010, 15, 1645-1667; doi:10.3390/molecules15031645

Article

\title{
Hitherto Unrecognized Fluorescence Properties of Coniferyl Alcohol
}

\section{Komandoor Elayavalli Achyuthan ${ }^{1,2, *}$, Paul David Adams ${ }^{1,3}$, Supratim Datta ${ }^{1,4}$, Blake Alexander Simmons ${ }^{1,4}$ and Anup Kumar Singh ${ }^{1,4}$}

1 Joint BioEnergy Institute (JBEI), Emeryville, CA 94550, USA

2 Biosensors and Nanomaterials Department, Sandia National Laboratories, Albuquerque, NM 87185, USA

3 Lawrence Berkeley National Laboratory, Berkeley, CA 94720, USA;

E-Mail: pdadams@lbl.gov (P.D.A.)

4 Sandia National Laboratories, Livermore, CA 94550, USA;

E-Mails: basimmo@sandia.gov (B.A.S.); sdatta2@lbl.gov (S.D.); aksingh@sandia.gov (A.K.S.)

* Author to whom correspondence should be addressed; E-Mails: kachyut@sandia.gov or

keachyuthan@lbl.gov; Tel.: +1-505-284-8979; Fax: +1-505-844-1198.

Received: 14 January 2010; in revised form: 23 February 2010 / Accepted: 8 March 2010 /

Published: 11 March 2010

\begin{abstract}
We instituted a quasi-quality assurance program for demonstrating coniferyl alcohol's fluorescence and fluorescence diminishment following enzymatic oxidation. The magnitude of diminishment was a measure of catalysis. High throughput screening was performed in pseudo-kinetic and endpoint modes by measuring the fluorescence at $416 \mathrm{~nm}$ following excitation at 290, 310 or $340 \mathrm{~nm}$. Dose-response tracings were linear between two and three orders of magnitude with average limits of detection and quantitation of 1.8 and $6.9 \mu \mathrm{M}$ coniferyl alcohol, respectively. Oxidation was evident with $0.025 \mu \mathrm{g} / \mathrm{mL}$ laccase or $0.003 \mu \mathrm{g} / \mathrm{mL}$ peroxidase or inside $5 \mathrm{~min}$ using $0.5 \mu \mathrm{g} / \mathrm{mL}$ laccase or $5 \mu \mathrm{M}$ substrate. Sodium chloride inhibited ( $\mathrm{IC}_{50}, 25 \mathrm{mM}$ ) laccase oxidation of coniferyl alcohol. Fluorescence from 10 concentrations ( 1 to $1000 \mu \mathrm{M}$ ) of coniferyl alcohol was stable for 24 hours over 14 excitation/emission cycles at 3 different combinations of excitation and emission wavelengths. In conclusion, coniferyl alcohol absorption and fluorescence assays should facilitate biomass lignin analyses and improve delignification.
\end{abstract}


Keywords: coniferyl alcohol; fluorescence; laccase; peroxidase; high throughput screening; lignin; biofuels

\section{Introduction}

Lignin is composed of three main phytochemicals: the monolignols of coniferyl alcohol (CA), sinapyl alcohol (SA) and $p$-coumaryl alcohol ( $p$-CA), that are cross-linked through $\beta-\mathrm{O}-4, \beta-1, \beta-5$, $\beta-\beta, 4-O-5$ and 5-5 bonds [1,2]. Depending on the plant species or tissue type, the monolignol content varies. Guaiacyl lignin of softwood (Gymnosperms), comprised principally of CA, is more resistant to delignification, whereas guaiacyl-syringyl lignin of hardwood (Angiosperms) containing CA and SA is easily degraded. Graminaceous lignin (grass) has high levels of $p$-CA [2]. Lignin composition is thus causally related to biomass recalcitrance, impeding the development of cost-effective biofuels [1-3]. Delignifying enzymes have not been identified in plants. However, filamentous white and brown rot fungi (Ascomycetes and Basidomycetes) degrade hardwood and softwood through phenoloxidative enzymes such as laccase and peroxidase [2]. For example, the white rot Basidomycetes Trametes versicolor degrades lignin through oxidative, demethoxylating and demethylating activities or via redox shuttle mediators (RSM) [2,4,5].

Knowledge of monolignol composition will allow genetic modifications for changing the lignin content towards reducing recalcitrance and enhancing saccharification [1,2]. Monolignol estimation might facilitate better pre-treatment strategies to enhance delignification [3]. We recently surveyed the methods for analyzing CA [6] and described high throughput screening (HTS) assays [7] for CA and p-cresol (both potential RSMs) [6,8,9]. Under a quasi-quality assurance (QA) program, we now describe hitherto unrecognized fluorescence properties of CA. We also provide a non-specialist primer on fluorescence in order to contextualize our findings. Our HTS assays utilized fluorescence changes due to laccase and peroxidase catalysis for detecting and quantitating CA oxidation. Our fluorescent assays are operable under pseudo-kinetic and endpoint modes with optical "turn off” signaling.

\section{Results and Discussion}

\subsection{CA Quality Assurance Program}

To our best information, the fluorescence properties of CA have not been described. We therefore instituted a quasi-QA program to demonstrate CA's fluorescence. We purchased CA from three independent vendors. Two of the sources were in geographically separate locations in the United States and the third was an overseas vendor. Absorption and fluorescence data were collected by two investigators, working independently at two different test sites, using three separate microplate spectrometers and two different enzymes (laccase and peroxidase) which used CA as the substrate. Similar experiments were carried out in two microwell density formats (96 or 384) using two different microplates (UV-transparent or white). The last metric was essential due to the potential for interference from the white plate's intrinsic fluorescence [10]. Tests conducted several weeks apart yielded similar profiles testifying to the reproducibility of the fluorescence properties of CA. We 
employed the well known absorption assays [6] (references cited therein) in order to uncover any differences amongst the CA samples from the three vendors before describing the fluorescence assays.

It is well known that CA absorption at $260 \mathrm{~nm}$ decreases following enzymatic oxidation [6 and references cited therein]. For the same $500 \mu \mathrm{M}$ concentration, in the absence of laccase, CA from Sigma-Aldrich had the highest absorbance in $\mathrm{pH} 4.5$ buffer (2.681 \pm 0.069 A.U), followed by CA from Acros Organics $(1.520 \pm 0.024)$, whilst CA from Alfa Aesar had the least absorbance $(0.675 \pm 0.004)$ (Figure 1A). The CA samples from Acros Organics and Alfa Aesar were rapidly oxidized with saturation between 0.25 to $1.0 \mu \mathrm{g} / \mathrm{mL}$ enzyme. On the other hand, CA from Sigma-Aldrich underwent gradual and linear oxidation over the 20 min reaction period with no evidence of saturation even at $\sim 1.0 \mu \mathrm{g} / \mathrm{mL}$ laccase. However, the saturation of the reaction velocity under acidic conditions is difficult to estimate due to the formation of light-scattering dehydrogenation polymers (DHPs) that transformed the reaction mixtures into cloudy suspensions [6].

Figure 1A. Laccase dose-response profiles for CA oxidation monitored by $260 \mathrm{~nm}$ absorption. Increasing concentrations of laccase were used to oxidize $500 \mu \mathrm{M}$ CA in $100 \mu \mathrm{L}$ of $\mathrm{pH} 4.5$ buffer under real-time kinetic measurements using UV-transparent 96-well microplate. Decreases in CA absorbance were measured at $20 \mathrm{~min}$ for the samples purchased from Alfa Aesar (open triangles, blue tracing), Acros Organics (open circles, red tracing) and Sigma-Aldrich (open squares, purple tracing).

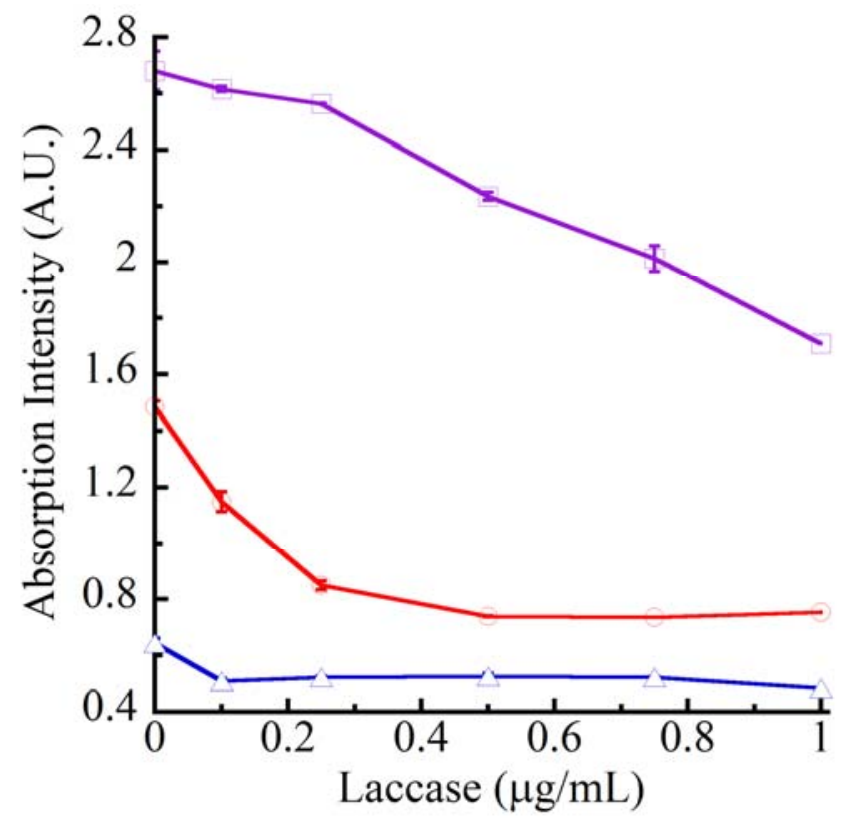

When laccase oxidation of CA samples was examined under basic $\mathrm{pH}$ conditions in endpoint mode at $60 \mathrm{~min}$ using the previously established $290 \mathrm{~nm}$ absorption wavelength [6], similar features were observed (Figure 1B). The DHPs appeared to dissolve entirely in base resulting in the reactions becoming clear and allowing the estimation of reaction velocity. In the absence of laccase, $500 \mu \mathrm{M}$ of CA from Sigma-Aldrich once again had the highest absorption in base (3.227 \pm 0.050 A.U.) followed by CA from Acros Organics (2.056 \pm 0.039 A.U.) and CA from Alfa Aesar trailing at $1.252 \pm 0.006$ A.U. As reported previously [6], CA absorption in $\mathrm{pH} 4.5$ buffer was lower than its absorption in base, 
regardless of the source. Similar to $\mathrm{pH} 4.5$ reactions, the CA from Sigma-Aldrich underwent a gradual oxidation compared to CA from Acros Organics or Alfa Aesar in endpoint mode (Figure 1B). The oxidation of CA in the 60 min endpoint reactions with $1.0 \mu \mathrm{g} / \mathrm{mL}$ laccase could be rank ordered as Sigma-Aldrich (58\%) > Acros Organics (43\%) > Alfa Aesar (18\%).

Figure 1B. Laccase dose-response profiles for CA oxidation monitored by $290 \mathrm{~nm}$ absorption. Increasing concentrations of laccase were used to oxidize $500 \mu \mathrm{M} \mathrm{CA}$ in $100 \mu \mathrm{L}$ of pH 4.5 buffer using UV-transparent 96-well microplate. At $60 \mathrm{~min}$, the reactions were stopped through basification (100 mM NaOH final). Decreases in absorbance were measured from the CA samples purchased from Alfa Aesar (open and closed triangles, blue tracings), Acros Organics (open and closed circles, red tracings) and Sigma-Aldrich (open and closed squares, purple tracings). Open symbols represent normalized percent decreases in the absorption intensity and closed symbols are the actual absorption units (A.U.).

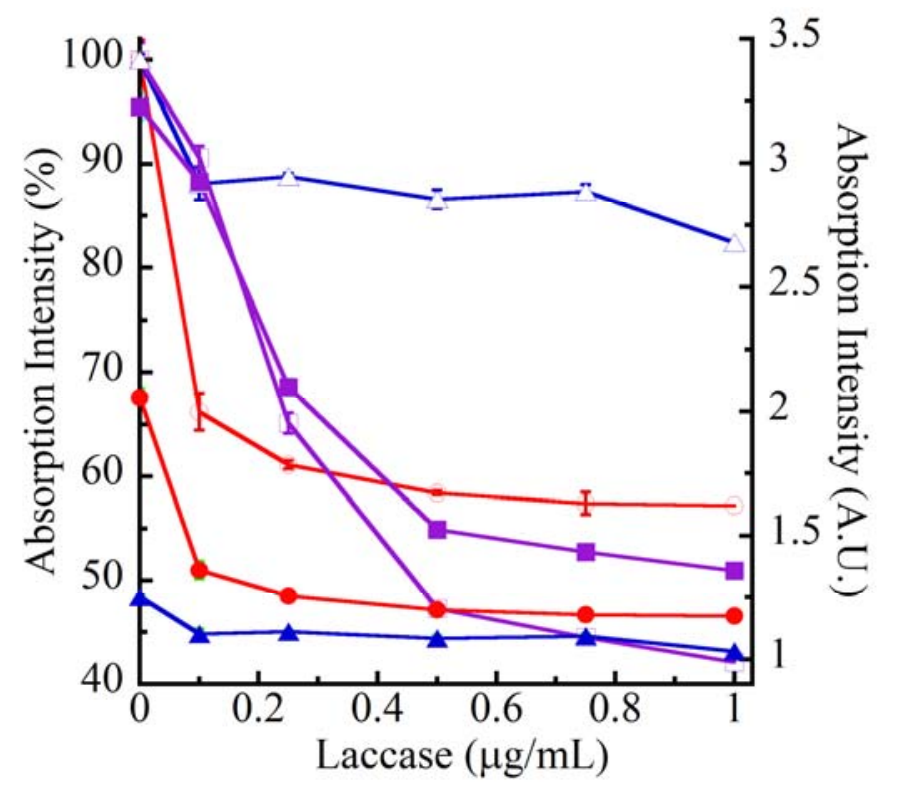

Kinetics of laccase-catalyzed oxidation of CA were executed in pseudo-kinetic mode with $0.1 \mu \mathrm{g} / \mathrm{mL}$ laccase and interrogated using $260 \mathrm{~nm}$ ( $\mathrm{pH} 4.5$ ) and $290 \mathrm{~nm}$ (base) absorption wavelengths. The absorbance values of the Sigma-Aldrich, Acros Organics and Alfa Aesar CA samples in pH 4.5 buffer (2.713 \pm 0.007 A.U., $1.649 \pm 0.100$ A.U. and $0.569 \pm 0.080$ A.U.) and in base (3.317 \pm 0.004 A.U., $2.434 \pm 0.066$ A.U. and $1.113 \pm 0.035$ A.U.) were similar to earlier values (Figure 1) confirming reproducibility. Reaction progress of Sigma-Aldrich and Acros Organics CA samples was discernible between 5 and $90 \mathrm{~min}$ with $0.1 \mu \mathrm{g} / \mathrm{mL}$ laccase. Alfa Aesar CA showed the least substrate activity in both pseudo-kinetic $(260 \mathrm{~nm})$ and endpoint $(290 \mathrm{~nm})$ absorption assays (Figure 2). However, the oxidation profiles of all CA samples were similar regardless of the interrogation wavelength or the $\mathrm{pH}$. 
Figure 2. Laccase kinetic (260 nm) and pseudo-kinetic (290 nm) oxidation of CA. Laccase $(0.1 \mu \mathrm{g} / \mathrm{mL})$ was used to oxidize $500 \mu \mathrm{M}$ CA in $100 \mu \mathrm{L}$ of $\mathrm{pH} 4.5$ buffer using UVtransparent 96-well microplate. Absorbance decrease from identical reactions was measured at $260 \mathrm{~nm}$ (open symbols) for the indicated time periods. Pseudo-kinetic reactions were stopped with base $(100 \mathrm{mM} \mathrm{NaOH})$ and absorbance decreases were measured at $290 \mathrm{~nm}$ (closed symbols). The CA samples analyzed were from Alfa Aesar (open and closed triangles, blue tracings), Acros Organics (open and closed circles, red tracings) and Sigma-Aldrich (open and closed squares, purple tracings).

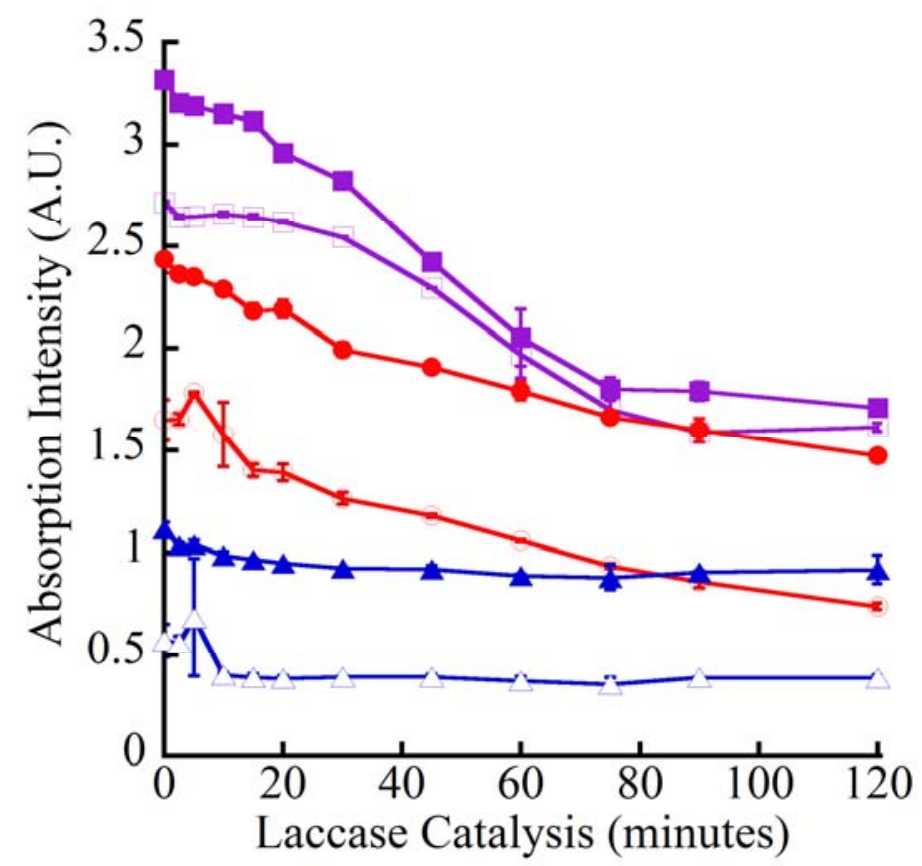

The absorption assays demonstrated that all three CA samples were oxidized by laccase to different extents depending on the source, with Sigma-Aldrich CA exhibiting the highest molar absorptivity and the most clearly delineated reaction kinetics with laccase. It would be speculation regarding the reasons for the differences between the CA samples and might be attributed to trace impurities in the CA from Alfa Aesar and to a lesser extent in the CA from Acros Organics. These contaminants might be quenching the absorption of CA and/or inhibiting the activity of the laccase. Interestingly, following basification of the laccase reactions, the mixtures containing CA from Acros Organics and Alfa Aesar turned pale yellow whereas similar reactions using Sigma-Aldrich CA remained colorless. The absorption assays showed that whereas all three CA samples could be used for investigating their fluorescence properties, the CA from Sigma-Aldrich might be the best choice. Therefore, during initial experiments we tested CA from all three vendors as part of our QA program, but later focused upon the Sigma-Aldrich CA for detailed fluorescence analyses.

\subsection{CA Absorption Spectra and Overlap with Excitation Spectra}

The absorption profiles of CA from Acros Organics and Alfa Aesar were similar to the absorption profile of Sigma-Aldrich CA (Figure 3), although as noted above their absorption intensities declined as follows: Sigma-Aldrich $>$ Acros Organics $>$ Alfa Aesar. The absorption maximum for all three CA 
samples was $290 \mathrm{~nm}$. All three absorption profiles spanned 250 to $350 \mathrm{~nm}$. The CA from SigmaAldrich had a broader peak compared to the sharper peaks of CA samples from Acros Oganics and Alfa Aesar. The Sigma-Aldrich CA absorption declined steeply resulting in a bell shaped curve (Figure 3, left panel, solid purple tracing). The declining profiles of CA from Acros Organics and Alfa Aesar were more gradual resulting in a squat shoulder, bathochromic to the absorption maximum (Figure 3, left panel, solid red and solid blue tracings).

We next scanned for CA excitation over the same wavelength range as its absorption spectrum and measured the emission at $420 \mathrm{~nm}$, thereby generating an excitation spectrum (Figure 3, left panel, broken tracings). Each CA's excitation peak was broad spanning $60 \mathrm{~nm}$ between 275 and $335 \mathrm{~nm}$. The excitation profiles of CA from the three vendors were nearly identical including the characteristic double excitation maxima composed of a smaller peak between 278 to $296 \mathrm{~nm}$ and a slightly larger peak at 304 to $324 \mathrm{~nm}$. The twin peaks were observed in multiple experiments over several days, in experiments conducted by different investigators at different locations, and were also observed in the excitation spectra of laccase-oxidized CA (Figure 4). Significant ( $80 \%$ of maximum) emission was noted between 280 to $330 \mathrm{~nm}$ excitation wavelengths (Figure 3). Similar to the absorption profiles, the three CA samples showed marked differences in their emission intensities declining as follows: SigmaAldrich $>$ Acros Organics $>$ Alfa Aesar. These results offered the first evidence for the fluorescence properties of CA and supported our QA model.

The overlapping profiles of the absorption and the excitation spectra (250 to $350 \mathrm{~nm}$ ) for all three CA samples were as expected. For aromatic compounds or compounds containing unsaturated bonds, the excitation spectrum is usually similar or identical to the absorption spectrum [11]. If we assume the quantum efficiency of fluorescence is independent of the excitation energy, then the signal intensity of the excitation spectrum is an indication of the variation in the photons absorbing energy as a function of the wavelength. Such was the case with the dilute $500 \mu \mathrm{M}$ solution of CA that was used to generate the absorption and excitation spectra. Therefore, the fraction of the exciting energy absorbed by CA is proportional to its absorption coefficient. Consequently, the excitation and absorption spectra of CA were overlapping (Figure 3). Spectral overlap also suggested the presence of a homogenous CA chromophore/fluorophore in solution. Although the excitation spectrum is often used to analyze the fluorescence of a mixture of absorbing compounds, in this case, the spectra allowed us to document the fluorescence properties of CA. Fluorescence assays are amongst the most widely used biochemical analysis tools and are regarded as the "gold standard" for detecting analytes [12].

\subsection{Emission and Excitation Spectra of CA}

The emission spectral profiles of CA samples from the three commercial sources were also nearly identical to one another (Figure 3, right panel). Consistent with the excitation spectral data, the CA from Acros Organics had lower emission intensity compared to Sigma-Aldrich CA but was higher than the fluorescence of CA from Alfa Aesar. Increasing concentrations of Sigma-Aldrich CA yielded progressively increasing fluorescence emission intensities between $1 \mu \mathrm{M}$ and $250 \mu \mathrm{M}$; at $500 \mu \mathrm{M}$ CA, the dose-response became negligible. The emission profile had maximum fluorescence intensity between 416 to $420 \mathrm{~nm}$ for all CA samples, a >100 nm Stokes shift, that avoided Rayleigh scattering (Figure 3). Nearly 75\% of maximum RFU was observed between the emission wavelengths of $412 \mathrm{~nm}$ 
to $446 \mathrm{~nm}$ with all samples. The $500 \mu \mathrm{M}$ Acros Organics CA had an emission profile overlapping the $25 \mu \mathrm{M}$ emission profile of CA from Sigma-Aldrich. Likewise, the emission profile of $500 \mu \mathrm{M}$ CA from Alfa Aesar was coincident with the emission profile of $10 \mu \mathrm{M}$ CA from Sigma-Aldrich. However, this does not mean that Sigma-Aldrich CA is 20- and 50-times more fluorescent on a molar basis compared to CA samples from Acros Organics and Alfa Aesar, respectively. It is clear from Figure 3 that the fluorescence intensity ceased to track linearly with CA concentration at $500 \mu \mathrm{M}$.

Figure 3. Absorption, excitation and emission spectra of CA. Left panel: Unmodified CA (100 $\mu \mathrm{L}$ of $500 \mu \mathrm{M} \mathrm{CA}$ in $100 \mathrm{mM} \mathrm{NaOH}$ ) was dispensed into a 96-well UV-transparent microplate. Absorption was monitored between 250 to $350 \mathrm{~nm}$ in $2 \mathrm{~nm}$ intervals (solid tracings) for CA purchased from Alfa Aesar (blue), Acros Organics (red) and SigmaAldrich (purple). Excitation spectral profiles (broken tracings) were monitored between 250 to $350 \mathrm{~nm}$ in $2 \mathrm{~nm}$ intervals (emission $=420 \mathrm{~nm}$ ) from CA purchased from Alfa Aesar (blue), Acros Organics (red) and Sigma-Aldrich (purple). The absorption and excitation profiles from $100 \mathrm{mM} \mathrm{NaOH}$ background are shown by the solid black tracing close to the abscissa. Right panel: The emission profiles of $100 \mu \mathrm{L}$ of CA in $100 \mathrm{mM} \mathrm{NaOH}$ ranged from 360 to $500 \mathrm{~nm}$ in $2 \mathrm{~nm}$ intervals with an excitation wavelength of $290 \mathrm{~nm}$. Fluorescence emission with increasing intensities represented by the solid tracings were from the following concentrations $(\mu \mathrm{M})$ of Sigma-Aldrich CA: 1.0 (magenta), 2.5 (green), 5 (maroon), 10 (grey), 25 (yellow), 50 (blue), 100 (aqua), 250 (red) and 500 (purple). The broken red and blue tracings represent the fluorescence emission from $500 \mu \mathrm{M}$ of CA from Acros Organics and Alfa Aesar, respectively. The black tracing close the abscissa is the background from $100 \mathrm{mM} \mathrm{NaOH}$.

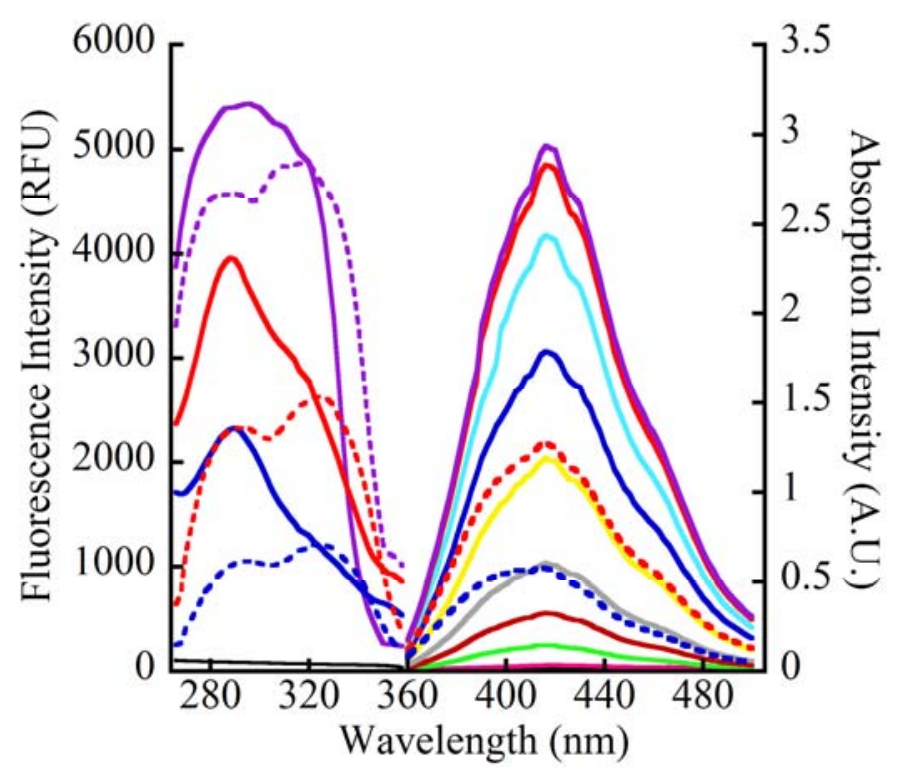

We empirically determined the excitation maximum by generating excitation spectra from 260 to $350 \mathrm{~nm}$ in $10 \mathrm{~nm}$ intervals (420 nm emission). Similar excitation maxima and excitation profiles were obtained using 96-well UV-transparent and white microplates by testing nine concentrations of Sigma-Aldrich CA (1 to $500 \mu \mathrm{M}$ ). Through these analyses, we concluded that 290 to $320 \mathrm{~nm}$ was the 
excitation maximum for CA. At flanking wavelengths, the emission intensity declined. For example, using 96-well white microplate and $270 \mathrm{~nm}$ excitation, the fluorescence of $250 \mu \mathrm{M}$ Sigma-Aldrich CA was 5837 RFU compared to 7819 RFU at $310 \mathrm{~nm}$ (25.3\% drop). Likewise, at the excitation wavelength of $330 \mathrm{~nm}$, the fluorescence was $6771 \mathrm{RFU}$ (13.4\% drop). Further out, the CA fluorescence dropped more steeply. At $260 \mathrm{~nm}$ excitation, the emission was $4353 \mathrm{RFU}$ (55.7\% of $\lambda \max$ ) and at $350 \mathrm{~nm}$ it was $2622 \mathrm{RFU}$ (33.5\% of $\lambda \max$ ). The higher fluorescence intensity of CA in white plate compared to the UV-transparent clear plate was due to light reflection from the white well walls. The data from the three samples supported our QA model and confirmed the fluorescence of CA.

The emission spectrum of CA, similar to most fluorophors, was red-shifted with respect to the absorption and excitation spectra. The excitation and emission spectra represent the probability distribution functions that a photon with particular quantum energy will be absorbed by CA. The excited state of CA loses the absorbed energy through the release of a second photon as fluorescence emission with lower energy and longer wavelength. This is because the fluorescence of CA is derived from the lowest vibrational level $(v=0)$ of the lowest electronically excited singlet state (S). The absorption of high-energy photons by CA is accompanied by energy loss through non-radiative processes before emission occurs. Consequently, the emitted photons have less energy compared to the absorbed photons, and the fluorescence is red-shifted relative to the absorption and excitation spectra (Figure 3). In compliance with Kasha's rule [11], CA displayed the same emission profiles irrespective of the excitation energy $(\lambda)$, although the emission intensity varied over these wavelengths (Figure 3 ). The identical emission spectra at different excitation wavelengths may be explained as follows. Upon excitation, CA enters a higher electronic and vibrational level and the excess energy is lost over picoto nano-second $\left(10^{-12}\right.$ to $\left.10^{-9}\right)$ lifetimes. CA drops to the lowest vibrational level of the first excited state (S1), from where emission takes place [11]. Due to the rapid relaxation lifetimes, the emission spectra of CA were identical at various excitation wavelengths and independent of the excitation energy. CA in solution does not display the detailed vibrational structure, resulting in the emission spectrum appearing as a broad band.

\subsection{Base Optimization}

The CA fluorescence was observed optimally under basic pH conditions. Either $100 \mathrm{mM} \mathrm{NaOH}$ or $250 \mathrm{mM} \mathrm{KOH}$ resulted in maximum fluorescence and S/B. For example, with $10 \mu \mathrm{M}$ of Sigma-Aldrich CA, using excitation wavelength of $310 \mathrm{~nm}$ and emission wavelength of $420 \mathrm{~nm}$, the fluorescence in $250 \mathrm{mM} \mathrm{KOH}$ was $1829 \pm 35 \mathrm{RFU}$ and the S/B was $3.42 \pm 0.10$. At flanking concentrations, both RFU and S/B declined. Thus, in $100 \mathrm{mM} \mathrm{KOH}$, the RFU and S/B were $1231 \pm 20$ and $2.23 \pm 0.06$, respectively and in $500 \mathrm{mM} \mathrm{KOH}$, these values were $1165 \pm 30 \mathrm{RFU}$ and S/B of $2.10 \pm 0.10$, respectively. Since the signal strengths were comparable in $100 \mathrm{mM} \mathrm{NaOH}$ or $250 \mathrm{mM} \mathrm{KOH}$, either base could be used. These results are in accordance with our fluorescence data of $p$-cresol [8] and hinted at similar ionization processes for both fluorophores. Furthermore, $100 \mathrm{mM} \mathrm{NaOH}$ or $250 \mathrm{mM}$ $\mathrm{KOH}$ was also optimal for monitoring the fluorescence changes following laccase- or peroxidaseoxidation of CA. 
Figure 4. Excitation and emission spectra of laccase-oxidized CA. Increasing concentrations of laccase were reacted with $250 \mu \mathrm{M}$ CA in $100 \mu \mathrm{L}$ of citrate buffer, $\mathrm{pH}$ 4.5. Reactions were carried out in 96-well UV-transparent microplate and stopped at 30 min through the addition of $250 \mathrm{mM} \mathrm{KOH}$. The $\mathrm{pH} 4.5$ reactions were cloudy due to the formation of DHPs. The cloudiness disappeared upon basification yielding clear solutions that were scanned over the excitation wavelengths of 250 to $350 \mathrm{~nm}$ in $2 \mathrm{~nm}$ intervals ( $\lambda$ em, $416 \mathrm{~nm}$ ) (left panel). The black tracing with the highest fluorescence is the excitation spectrum of $250 \mu \mathrm{M}$ unmodified CA. The profiles with decreasing fluorescence intensities represent the excitation spectra of CA oxidized by 0.01 (blue), 0.05 (red), 0.1 (green) and 0.25 (purple) $\mu \mathrm{g} / \mathrm{mL}$ laccase. Samples were then excited using $310 \mathrm{~nm}$ wavelength and the emission was scanned between 360 and $500 \mathrm{~nm}$ in $2 \mathrm{~nm}$ intervals (right panel). The black tracing with the highest fluorescence intensity is the emission spectrum of $250 \mu \mathrm{M}$ unmodified CA. The tracings with progressively declining fluorescence intensities represent the emission spectra of CA oxidized using 0.01 (blue), 0.05 (red), 0.1 (green) and 0.25 (purple) $\mu \mathrm{g} / \mathrm{mL}$ laccase.

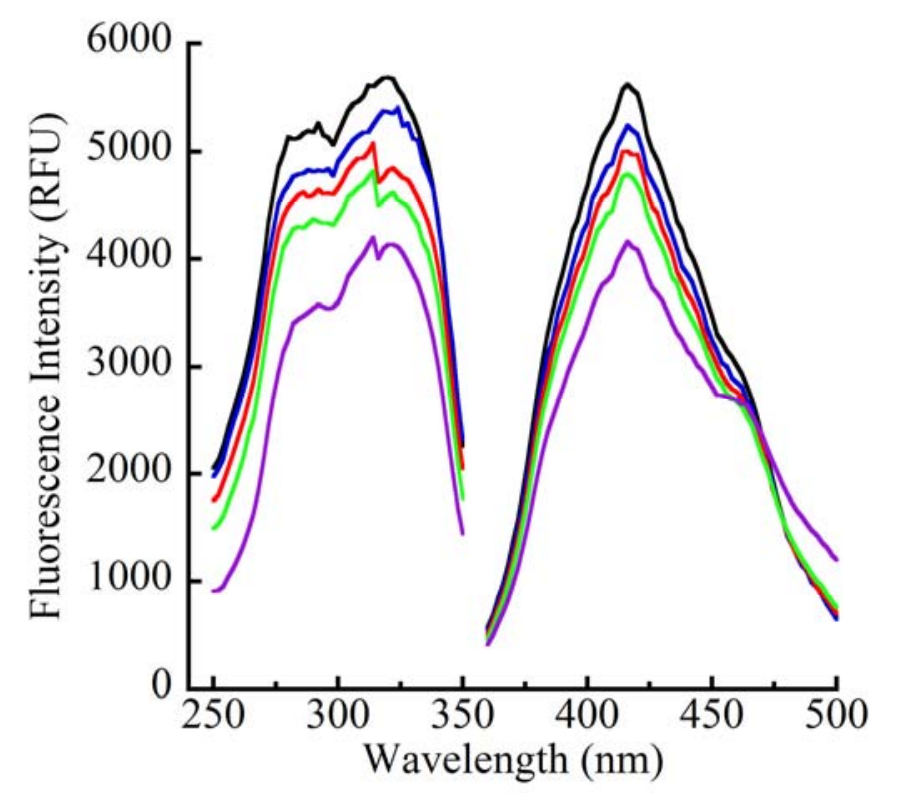

\subsection{Excitation and Emission Spectra of Laccase-Oxidized CA}

Laccase oxidation diminished the fluorescence of CA with the magnitude of diminishment increasing between 0.01 to $0.25 \mu \mathrm{g} / \mathrm{mL}$ concentrations of laccase as seen in both the excitation and emission sweeps (Figure 4). However, the excitation and emission profiles of oxidized CA reactions were identical to the corresponding profiles of the unmodified CA (Figure 3). This indicated that we were monitoring the fluorescence of CA during catalysis and not the fluorescence of its oxidation products. Fluorescence decrease following oxidation might reflect a transformation of the CA into non-fluorescent products. This will result in lower levels of residual unmodified CA being present in the reaction mixtures, leading to diminished fluorescence but retaining the same excitation and emission profiles of unmodified CA. The data do not exclude a quench effect of the oxidation products on CA's fluorescence. Whether CA oxidation products (Scheme 1) are fluorescent or otherwise quench 
the fluorescence of unmodified CA is a topic for future work. This involves isolating the products, verifying structure and purity and testing each product for fluorescence and/or quench properties. This is beyond the scope of our current work where we are limiting to the demonstration of the hitherto unrecognized fluorescence properties of CA.

Scheme 1. Chemical structures of CA and its main oxidation products. The various compounds shown are: coniferyl alcohol or CA (I), $\beta-5$ dehydrodimer or dehydrodiconiferyl alcohol (II), $\beta$-O-4 dehydrodimer or guaiacylglycerol $\beta$-O-4 coniferyl alchol ether (III) and $\beta$ - $\beta$-dehydrodimer or pinoresinol (IV). It should be noted that compound III occurs in two different diastereoisomeric configurations of erythroguaiacylglycerol- $\beta$-coniferyl ether and threo-guaiacylglycerol- $\beta$-coniferyl ether. In addition to these compounds, the so called dehydrogenation polymers (DHPs) and oligomers might also be formed depending upon the catalytic conditions such as the type and amount of enzyme (laccase or peroxidase) used, the duration of catalysis, reaction temperature, the concentration of the substrate, and other conditions.
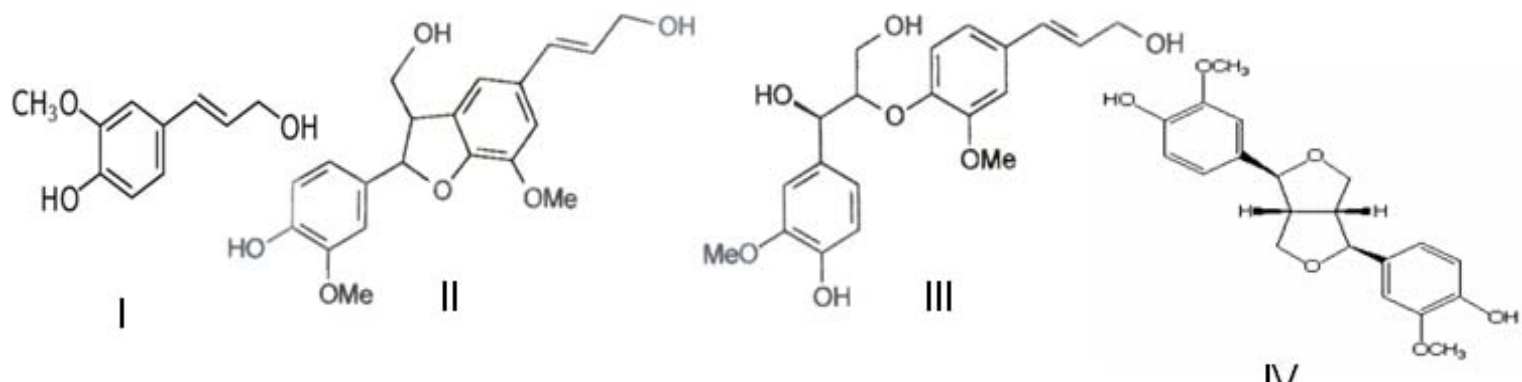

\subsection{Oxidation Kinetics of $C A$}

Similar to laccase reactivity of CA samples monitored by absorption spectroscopy (Figures 1 and 2), the reaction profiles tracked in a like manner when examined by fluorescence spectroscopy (Figure 5). Thus, CA from Alfa Aesar had the smallest decline in fluorescence intensity consequent to laccase catalysis and the velocity saturated within $30 \mathrm{~min}$. On the other hand, laccase oxidation of CA from Acros Organics and Sigma-Aldrich proceeded more gradually. Sigma-Aldrich CA displayed the greatest linearity of velocity over time. The oxidation profiles of Sigma-Aldrich CA also tracked closely whether analyzed by fluorescence decline or percent oxidation (Figure 5). The percent oxidation at 60 min was the greatest with Sigma-Aldrich CA (82\%) followed by Acros Organics (37\%) and trailed by Alfa Aesar (19\%). These percent oxidations were remarkably close to the values determined in the absorption assay (Figure 1B) especially with CA from Acros Organics and Alfa Aesar. The higher percent oxidation of Sigma-Aldrich CA in the fluorescence assay (Figure 5) might be due to its greater sensitivity particularly for the better performing substrate. Reactivity of CA from the three vendors could be rank ordered as Sigma-Aldrich > Acros Organics > Alfa Aesar. The same trend lines were observed when the reactions were interrogated using 310nm and $340 \mathrm{~nm}$ excitation wavelengths. Absorption and fluorescence spectroscopic data were consistent for all CA samples, thus validating our QA model. 
Figure 5. Laccase pseudo-kinetic oxidation of CA. Laccase $(0.1 \mu \mathrm{g} / \mathrm{mL})$ was used to oxidize $500 \mu \mathrm{M}$ CA in $\mathrm{pH} 4.5$ buffer in 96-well UV-transparent microplate. Identical reaction sets were stopped at the indicated time points using base (100 $\mathrm{mM} \mathrm{NaOH})$. The CA samples were then excited using $290 \mathrm{~nm}$ wavelength and the emission was monitored at $416 \mathrm{~nm}$. The CA samples analyzed were purchased from Alfa Aesar (open and closed triangles, blue tracings), Acros Organics (open and closed circles, red tracings) and SigmaAldrich (open and closed squares, purple tracings). Open symbols represent percent decrease in fluorescence intensity and closed symbols are fluorescence intensities (RFU).

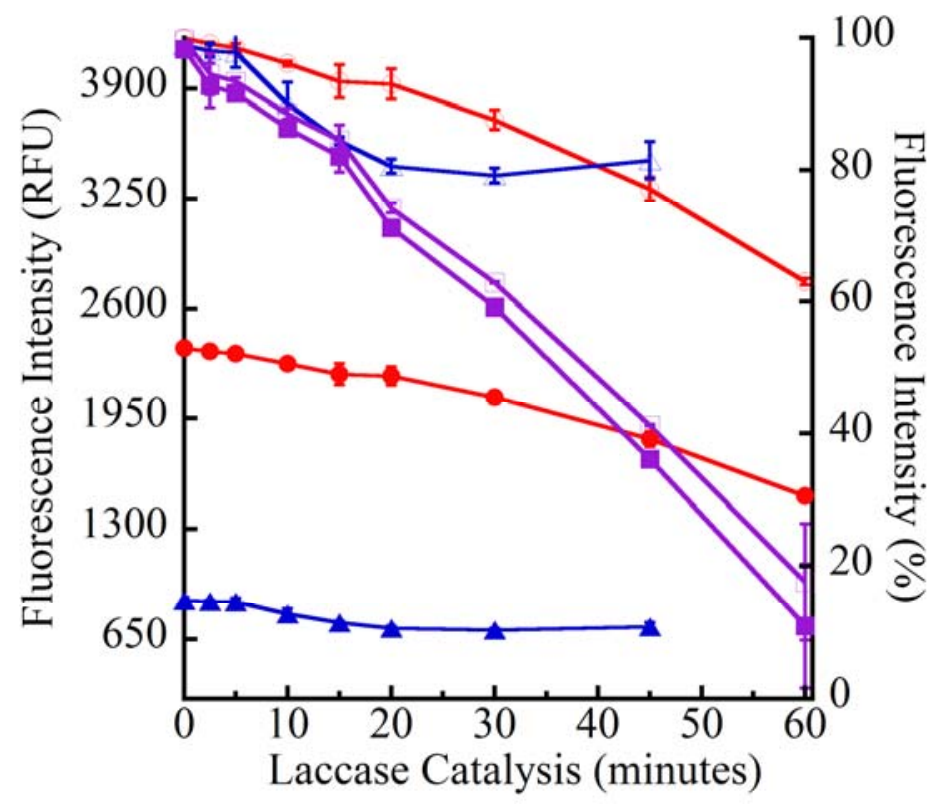

\subsection{Reproducibility of CA Fluorescence}

Excitation and emission profiles identical to those shown in Figures 3 and 4 were generated using CA from all three vendors, when the measurements were carried out by two different investigators working in different locations and using different microplate fluorometers. The CA sample from Sigma-Aldrich exhibited the highest dose-response relationship compared to CA of Acros Organics and Alfa Aesar. For example, in 96-well UV-transparent microplate, 250 and $500 \mu \mathrm{M}$ of CA from Alfa Aesar yielded fluorescence intensities of $572 \pm 38$ and $843 \pm 26$ RFU compared to 4875 RFU from $250 \mu \mathrm{M}$ CA from Sigma-Aldrich; greater than 10-fold difference. The CA samples from SigmaAldrich, Acros Organics and Alfa Aesar all displayed the characteristic fluorescence decrease following oxidation by laccase. However, the magnitude of fluorescence diminishment was the highest and therefore the $\triangle$ RFU values the largest, with CA from Sigma-Aldrich. Since both RFUs and S/B were highest with CA from Sigma-Aldrich, we used this reagent for the remainder of the experiments.

\subsection{Laccase Dose-Response}

The dose-response profiles were similar when the $\Delta$ RFUs were tracked for 0.01 to $10 \mu \mathrm{g} / \mathrm{mL}$ enzyme and the reaction velocity saturated between $\sim 0.5$ to $10 \mu \mathrm{g} / \mathrm{mL}$ enzyme at all excitation wavelengths (Figure 6). However, the magnitude of the signal discrimination depended on the excitation wavelength with the $\Delta$ RFUs from 290 and $310 \mathrm{~nm}$ being similar and larger than the $\Delta$ RFUs 
obtained with $340 \mathrm{~nm}$ that in turn was greater than the $\Delta$ RFUs with $360 \mathrm{~nm}$. The $\Delta$ RFUs became progressively larger with increasing concentrations of laccase due to the increasing fluorescence diminishment following CA oxidation. The use of base prior to fluorescence spectroscopy also stopped the enzymatic oxidation "instantaneously" due to laccase denaturation and by shifting drastically from the optimum $\mathrm{pH}$ for laccase [8]. Thus, laccase oxidation of CA could be monitored in pseudo-kinetic or endpoint mode by fluorescence spectroscopy (Figures 4-6). The lag phase in the reaction velocity observed in Figure 6 might be due to the logarithmic increase in the concentration of the enzyme.

Figure 6. Laccase dose-response for CA oxidation. Increasing concentrations of laccase were used to oxidize $500 \mu \mathrm{M}$ CA for $60 \mathrm{~min}$. Reactions were carried out in 96-well white plate (100 $\mu \mathrm{L} /$ well) and stopped using $250 \mathrm{mM} \mathrm{KOH}$ (endpoint mode). Fluorescence was measured at $416 \mathrm{~nm}$ after exciting the samples using $290 \mathrm{~nm}$ (open squares, purple tracing), $310 \mathrm{~nm}$ (closed squares, red tracing), $340 \mathrm{~nm}$ (open circles, blue tracing) and $360 \mathrm{~nm}$ (closed circles, black tracing).

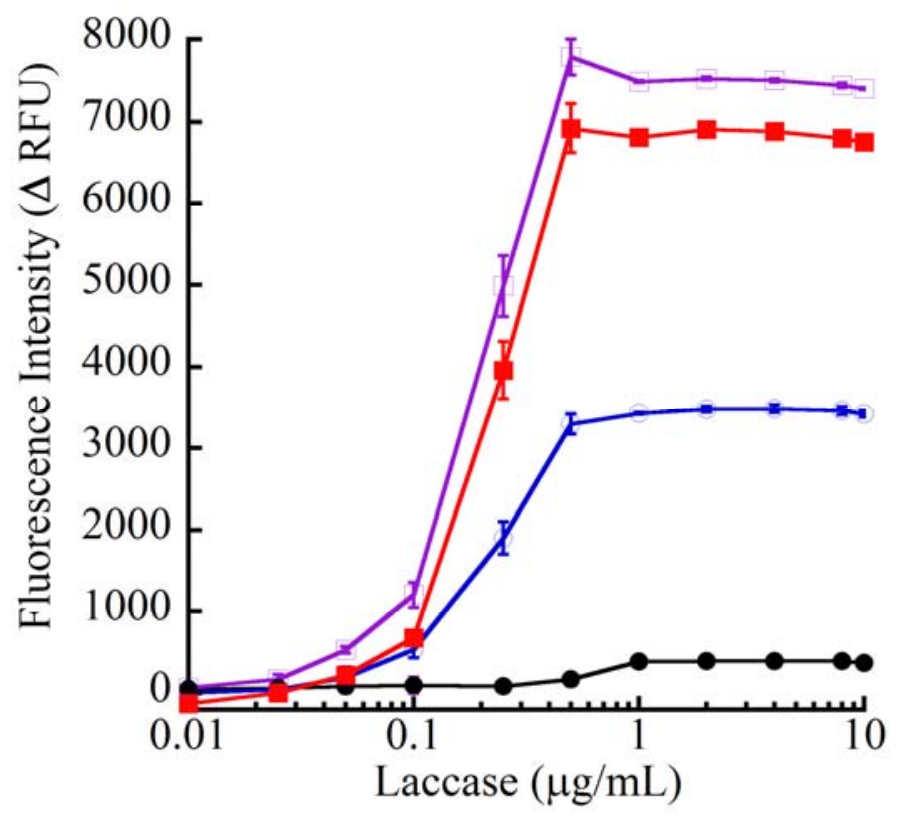

\subsection{Kinetics of Laccase- and Peroxidase-Catalyzed CA Oxidation}

Peroxidase is a major enzyme along with laccase influencing lignin biosynthesis and degradation $[2,5]$. In order to expand the fluorescence spectroscopic analyses of CA, we monitored the $\Delta$ RFU trajectories of laccase- and peroxidase-oxidized CA in pseudo-kinetic mode since progress curve is an indicator of enzyme-catalyzed reactions [13]. We also used these results to demonstrate our $\Delta$ RFU analyses in a graphical format (Figure 7). The peroxidase and laccase assays were done using different microplate readers located at two different test sites. The fluorescence intensity of CA in the absence of peroxidase (7930 $\pm 55 \mathrm{RFU} ; n=24$; <1\% error) or laccase (10647 $\pm 131 \mathrm{RFU} ; n=18$; <2\% error) underwent little change over the 60 to $90 \mathrm{~min}$ reaction period (Figure 7). Differences in the fluorescence intensities of the CA could be due to instrument variation at the two test locations. Parenthetically, this data provided the first hint of CA stability. Laccase or peroxidase oxidation of CA led to progressively diminishing fluorescence intensity over time as seen by the tracings with the 
negative slopes (Figure 7). On the other hand, the $\Delta$ RFUs had an upward trajectory. Laccase and peroxidase catalysis displayed first order reaction rate with linear trend lines between 2.5 and 30 min (peroxidase) or $60 \mathrm{~min}$ (laccase), indicating that the formation of oxidation products was proportional to the catalysis time. The rapid saturation of velocity at a lower concentration of peroxidase might indicate a greater reactivity of this enzyme towards CA relative to laccase (Figure 7). The data showed that laccase or peroxidase oxidation of CA could be detected within $5 \mathrm{~min}$.

Figure 7. Pseudo-kinetics of laccase- and peroxidase-catalyzed oxidation of CA. Laccase $(0.5 \mu \mathrm{g} / \mathrm{mL})$ or peroxidase $(0.1 \mu \mathrm{g} / \mathrm{mL})$ was reacted with $250 \mu \mathrm{M}$ CA in 96 -well white plates for the indicated duration and then stopped with $250 \mathrm{mM} \mathrm{KOH}$. Fluorescence was measured using $\lambda$ ex of 290, 310 and $340 \mathrm{~nm}$ with $\lambda$ em of $416 \mathrm{~nm}$. Due to the nearly identical kinetic profiles, only the data from $\lambda$ ex of $290 \mathrm{~nm}$ is shown. The black tracings are "control” fluorescence from unmodified CA during peroxidase (closed triangles) or laccase (open triangles) catalysis. Closed circles (red dotted tracing) represent $\Delta$ RFU from peroxidase oxidized CA with linearity $\left(r^{2}\right)$ between 2.5 and 30 min of 0.978 (solid purple curve fit). Open circles (solid red tracing) are decreasing emission intensities over time following peroxidase catalysis. Open squares connected by the solid blue tracing represent fluorescence decline due to laccase oxidation of CA. Closed squares connected by dotted blue tracing are the upward trajectory of $\Delta$ RFU values from laccase-oxidized CA. Linearity $\left(r^{2}\right.$; solid green tracing) was 0.997.

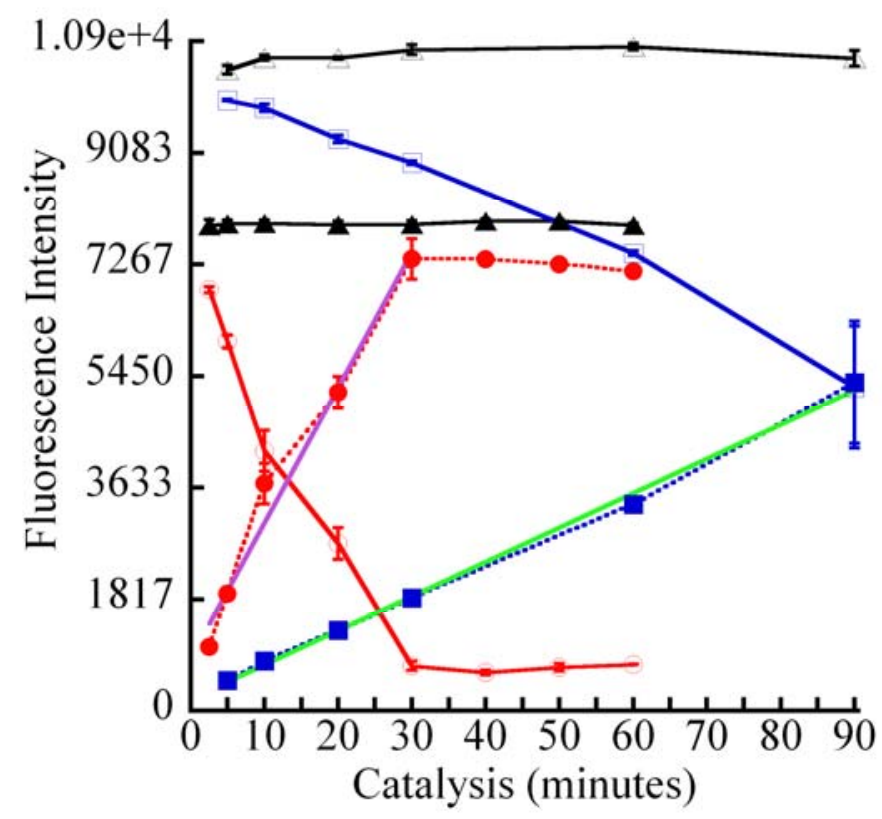

Laccase is an important enzyme secreted by white rot Basidomycetes such as Trametes versicolor $[2,4,5]$, the fungal source of our enzyme. The demonstration of physiologically-relevant substrates such as CA is useful for understanding the role of these phenoloxidative enzymes during delignification $[2,4,5]$. Finally, the data demonstrated that even though highly basic conditions were required for eliciting fluorescence from unmodified and oxidized CA, the assay was still useful for determining the oxidation kinetics through pseudo-kinetic analysis (Figures 7). Basification instantaneously stopped the catalysis at the desired time point and also facilitated maximum emission. 
With robotics and liquid handling systems, these steps can be integrated and automated for HTS [7]. The endpoint and pseudo-kinetic analyses conferred flexibility to our fluorescence assays of CA and its oxidation by laccase or peroxidase.

\subsection{Dose-Response for Peroxidase Oxidation of CA}

Similar to laccase, the magnitude of CA's fluorescence decrease was related to peroxidase catalysis duration (Figure 7) or peroxidase concentration (Figure 8). The dose-response tracings showed a linear (correlation of coefficient, $r^{2} \geq 0.90$ ) increase in reaction velocity at low concentrations (up to $50 \mathrm{ng} / \mathrm{mL}$ peroxidase) of enzyme followed by zero order at higher concentrations (50 to $200 \mathrm{ng} / \mathrm{mL}$ enzyme) at all excitation wavelengths examined (Figure 8). Depending on the reaction conditions, as little as $2.5 \mathrm{ng} / \mathrm{mL}$ peroxidase was sufficient to elicit a measurable $\Delta \mathrm{RFU}$. This further confirmed the fluorescence properties of CA. Since laccase and peroxidase oxidation diminished the fluorescence, both enzymes might be acting promiscuously upon CA. It is likely that the reaction products from both catalysts might be the same or similar, based upon their absorption and fluorescence responses [6].

Figure 8. Peroxidase-catalyzed CA oxidation. Increasing concentrations of horseradish peroxidase were used to oxidize $250 \mu \mathrm{M}$ CA in the presence of $250 \mu \mathrm{M} \mathrm{H}_{2} \mathrm{O}_{2}$ for 30 min using 96-well UV-transparent microplate. The reaction was stopped using $100 \mathrm{mM} \mathrm{NaOH}$ and the fluorescence was measured at $416 \mathrm{~nm}$ following excitation using 290 (open squares, purple), 310 (closed squares, red) and $340 \mathrm{~nm}$ (open circles, blue) wavelengths. Dotted tracings are interpolations and solid tracings are the linear curve fit (5 to $40 \mathrm{ng} / \mathrm{mL}$ peroxidase). Linearity $\left(r^{2}\right)$ values were 0.999 (290 nm, solid purple), 0.999 (310 nm, solid red) and 0.903 (340 nm, solid blue).

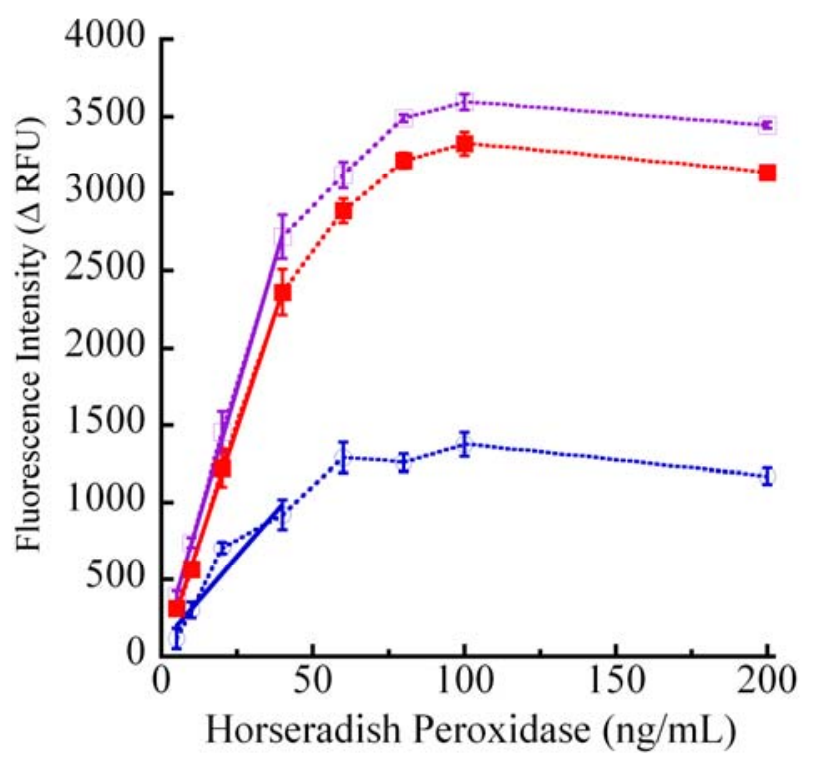

\subsection{High Throughput Screening (HTS) Optimization}

A goal of our work was to establish HTS conditions for the analyses of biofuel-critical phytochemicals such as CA. The advantages of HTS assays are speed, sample conservation due to 
small reaction volumes, lower cost, less waste, shorter turnaround of data, automation for unattended, $24 \times 7$ operations, and the ability to screen hundreds of thousands of samples every day. Paired with bioinformatics, lignin compositional analyses can be rapidly connected to the best pre-treatment strategy for improving saccharification.

The fluorescence emission profiles of CA were nearly identical when the reactions were carried out using UV-transparent or opaque white plates. Fluorescence intensity and S/B were approximately double with white plates due to light reflectance from the well walls. The reaction volumes could be varied from $25 \mu \mathrm{L}$ (384-wells) to $300 \mu \mathrm{L}$ (96-wells) for measuring unmodified CA as well as laccaseor peroxidase-oxidized CA. These data are useful for transitioning our assays to ultra-HTS (1536wells) with robotics-aided liquid handling, guided by the design considerations described before [7]. One aspect of HTS is inhibitor evaluation of compound libraries. We therefore investigated halide inhibition of laccase oxidation of CA as a model for compound library screening [8].

Figure 9. Halide inhibition of laccase-catalyzed CA oxidation. Indicated concentrations of $\mathrm{NaCl}$ were added either during the enzymatic reactions (closed circles, red tracing) or after stopping the reactions with $250 \mathrm{mM} \mathrm{KOH}$ (open circles, blue tracing). The effect of $\mathrm{NaCl}$ on the fluorescence of unmodified CA is shown by the purple tracing connecting the closed squares. Nearly identical profiles were generated by exciting the samples at 290, 310 and $340 \mathrm{~nm}$ and measuring the emission at $416 \mathrm{~nm}$. The data shown in this figure were obtained by excitation of CA at $290 \mathrm{~nm}$.

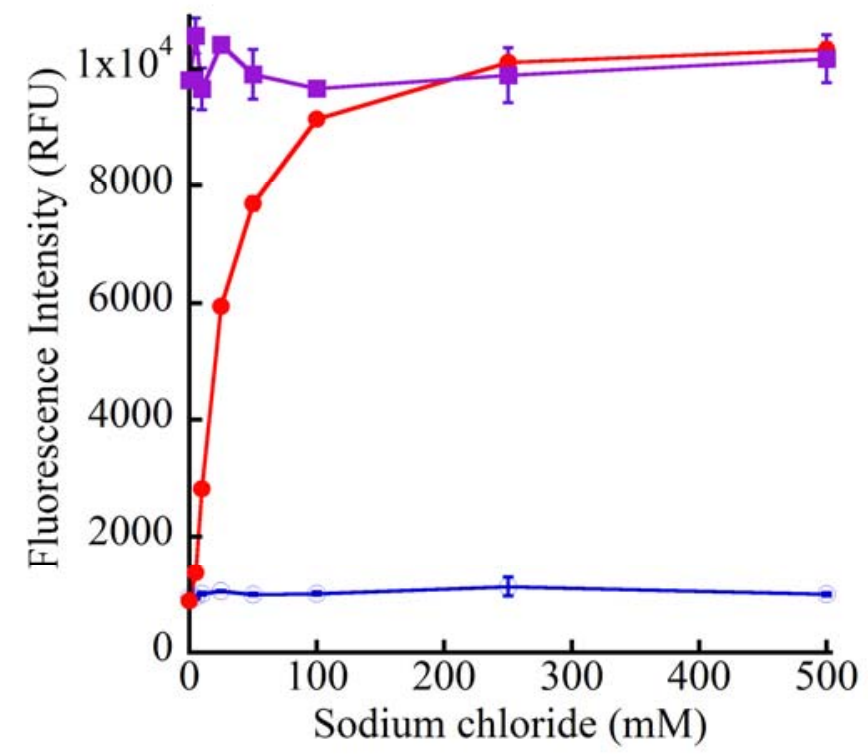

Halides bind to the copper atoms of fungal laccases which interrupts the electron shuttle and thereby inactivates the enzyme. Laccase inhibition profiles by halides are complex and calculation of the $\mathrm{IC}_{50}$ values might be adequate to document inhibition of laccase-catalyzed CA oxidation [14]. In agreement with our previous data of laccase inhibition of $p$-cresol oxidation [8], an $\mathrm{IC}_{50}$ of $\sim 25 \mathrm{mM}$ $\mathrm{NaCl}$ was calculated for the laccase inhibition of CA oxidation (Figure 9). With increasing concentrations of $\mathrm{NaCl}$ laccase is progressively inhibited, leading to the accumulation of unmodified CA resulting in increased fluorescence (Figure 9, red). Salt had no effect on the residual fluorescence of laccase-oxidized CA or unmodified CA fluorescence (Figure 9, blue and purple tracings). These 
data provide a basis for the HTS of laccase inhibitors using $\mathrm{NaCl}$ as an exemplar [8]. Reversibility of fluorescence diminishment due to laccase inhibition also affirmed the fluorescence properties of CA.

\subsection{CA Fluorescence Sensitivity}

Consistent with the data of Figure 3, the LOD and LOQ of Sigma-Aldrich CA were determined to be 1.8 and $6.9 \mu \mathrm{M}$, respectively (288 ppb and $1.2 \mathrm{ppm}$ ) (Table 1). We could detect and quantify 40 and 170 pmol respectively, of CA in $25 \mu \mathrm{L}$ reactions using 384-well plate.

Table 1. Limits of detection (LOD) and quantitation (LOQ) of CA.*

\begin{tabular}{|c|c|c|c|c|c|}
\hline $\begin{array}{c}\text { Excitation } \\
\text { wavelength } \\
(\boldsymbol{\lambda})\end{array}$ & $\begin{array}{c}\text { LOD } \\
(\boldsymbol{\mu M})\end{array}$ & $\begin{array}{c}\text { LOD } \\
(\mathbf{p p b})\end{array}$ & $\begin{array}{c}\text { LOQ } \\
(\boldsymbol{\mu M} \mathbf{M})\end{array}$ & $\begin{array}{c}\text { LOQ } \\
(\mathbf{p p m})\end{array}$ & $\begin{array}{c}\text { Linearity } \\
\left.\boldsymbol{(}^{2}\right)\end{array}$ \\
\hline $290 \mathrm{~nm}$ & 1.5 & 263 & 6.5 & 1.2 & 0.989 \\
$310 \mathrm{~nm}$ & 1.5 & 259 & 7.7 & 1.4 & 0.970 \\
$340 \mathrm{~nm}$ & 2.5 & 435 & 6.7 & 1.2 & 0.910 \\
\hline
\end{tabular}

* Fluorescence from CA solutions in $250 \mathrm{mM} \mathrm{KOH}$ was measured at $416 \mathrm{~nm}$.

The LOD and LOQ were not different at excitation wavelengths of 290 and $310 \mathrm{~nm}$. However, with $340 \mathrm{~nm}$ excitation the LOD was $2.5 \mu \mathrm{M}$ CA. Fluorescence intensity was similar for 25 and $50 \mu \mathrm{L}$ reactions with excitation wavelength of 290 or $310 \mathrm{~nm}$ (Figure 10A).

Figure 10A. Sensitivity of CA. Increasing $(1.0$ to $1000 \mu \mathrm{M})$ concentrations of unmodified CA diluted in $100 \mathrm{mM} \mathrm{NaOH}$ were dispensed into a 384-well white plate as 25 or $50 \mu \mathrm{L}$ solutions. Fluorescence from the samples was measured at $416 \mathrm{~nm}$ using the excitation wavelengths of 290 (purple tracings; open squares, $25 \mu \mathrm{L}$; closed squares, $50 \mu \mathrm{L}$ ), 310 (red tracings; open triangles, $25 \mu \mathrm{L}$; closed triangles, $50 \mu \mathrm{L}$ ) and $340 \mathrm{~nm}$ (blue tracings; open circles, $25 \mu \mathrm{L}$; closed circles, $50 \mu \mathrm{L}$ ).

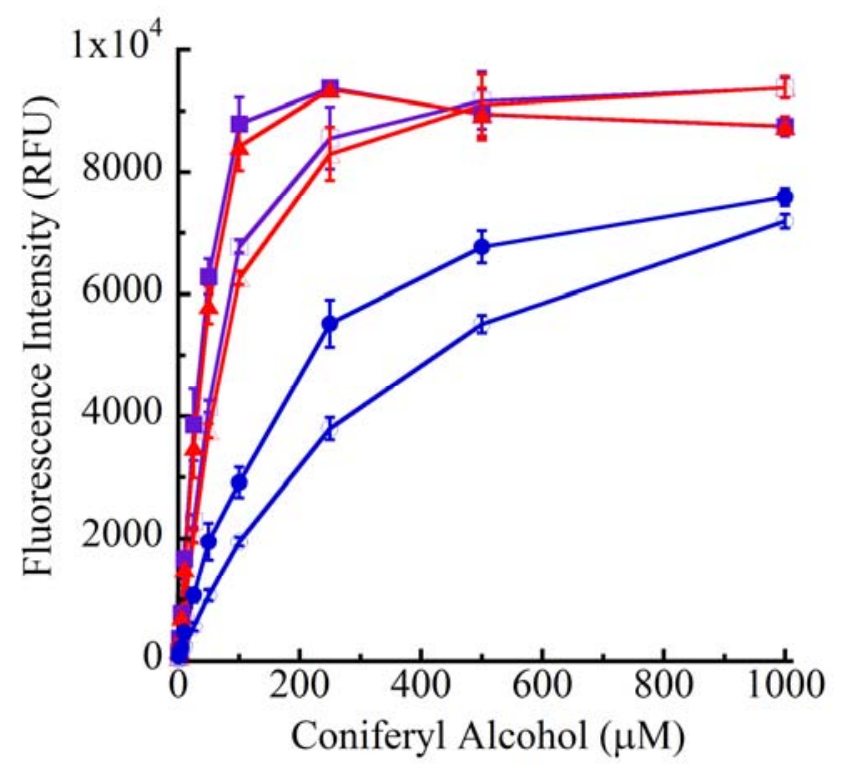


Although the emission intensities were less discriminating at these wavelengths for 25 and $50 \mu \mathrm{L}$ reactions, and the molar concentration of CA were identical, the amount of CA was double in $50 \mu \mathrm{L}$ reactions compared to $25 \mu \mathrm{L}$. With $340 \mathrm{~nm}$ excitation, the emission became less intense for the $25 \mu \mathrm{L}$ reactions relative to the $50 \mu \mathrm{L}$ reactions (Figures 10A and $\mathrm{B}$ ). All dose-response tracings were linear $\left(r^{2}>0.95\right)$ over two orders of magnitude of CA concentrations (1 to $\left.100 \mu \mathrm{M}\right)$ and the signals reached a plateau thereafter (Figures 10A and B). These calculations were reproducible when the CA was retested more than two weeks later.

A final point to note is that the same set of CA samples after absorption measurements in base [6] could be used for fluorescence measurements offering assay simplicity, ease and flexibility. Additional assay simplification might be achieved by carrying out the enzymatic and/or non-enzymatic reactions in the desired well volume at $\mathrm{pH} 4.5$ using UV-transparent clear plate. After interrogation of the samples by absorption spectroscopy [6], concentrated base can be added such that the volume change is $<5 \%$. Finally, the samples can be re-interrogated using endpoint absorption [6, present work], pseudo-kinetic and endpoint fluorescence spectroscopy (present work). Thus, a single set of samples could be interrogated using multimode spectroscopy under real-time kinetic, pseudo-kinetic and endpoint modes, under acidic or basic conditions.

Figure 10B. Linearity of CA Dose-Response. All conditions are described in Figure 10A. The $r^{2}$ values were: $0.984(\lambda \mathrm{ex}=290 \mathrm{~nm}, 25 \mu \mathrm{L}$; open squares, purple); 0.945 $(\lambda \mathrm{ex}=290 \mathrm{~nm}, 50 \mu \mathrm{L}$; closed squares, purple); $0.989(\lambda \mathrm{ex}=310 \mathrm{~nm}, 25 \mu \mathrm{L}$; open triangles, red); 0.958 ( $\lambda \mathrm{ex}=310 \mathrm{~nm}, 50 \mu \mathrm{L}$; closed triangles, red); 0.997 ( $\lambda \mathrm{ex}=340 \mathrm{~nm}, 25 \mu \mathrm{L}$; open circles, blue) and 0.973 ( $\lambda \mathrm{ex}=340 \mathrm{~nm}, 50 \mu \mathrm{L}$; closed circles, blue).

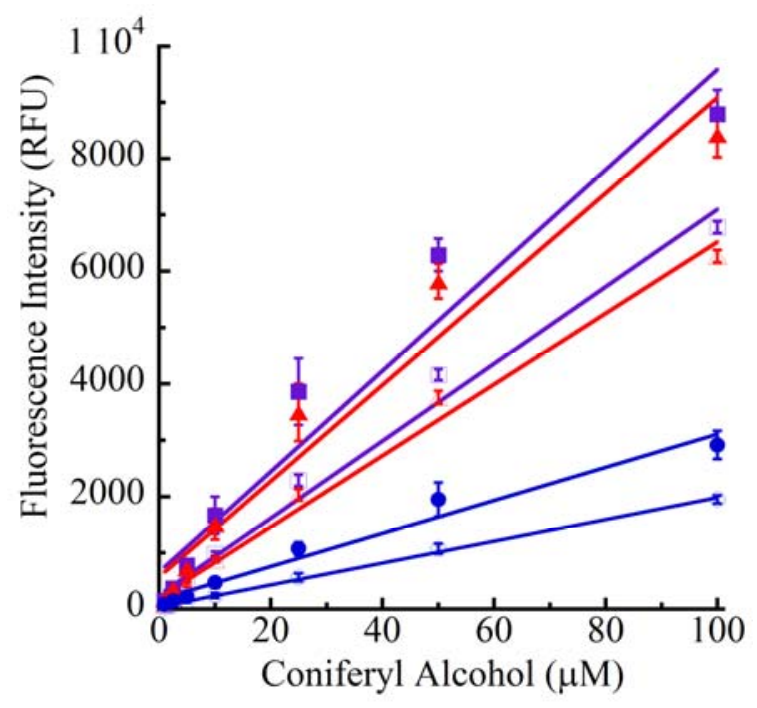

\subsection{CA Fluorescence Stability}

The certificate of analysis from three different vendors commented on the susceptibility of CA to air and light oxidation. We therefore studied the photostability, photodegradation and photobleaching of Sigma-Aldrich CA's fluorescence. Since identical stability profiles were generated under all interrogation wavelengths and all the data were mutually consistent, only the data obtained with 310 nm excitation and $25 \mu \mathrm{L}$ volume are presented in Figures 11 and 12. The data demonstrated 
photostability of CA over three orders of concentrations $(1 \mu \mathrm{M}$ to $1000 \mu \mathrm{M})$ for 24 hours at $\sim 25{ }^{\circ} \mathrm{C}$ (Figures 11A and B).

Figure 11A. Concentration dependence of CA photostability. Increasing concentrations of CA were dispensed into 384-well microplate. The data from the $25 \mu \mathrm{L}$ reactions ( $\lambda$ ex, $310 \mathrm{~nm} ; \lambda$ em $=416 \mathrm{~nm}$ ) are shown. The tracings represent measurements taken at the following intervals (min): 5 (black), 10 (blue), 20 (red), 30 (purple), 45 (bright green), 60 (yellow), 120 (aqua), 180 (maroon), 240 (dark grey), 300 (magenta), 360 (brown), 420 (orange), 480 (dark green) and 1440 (light grey).

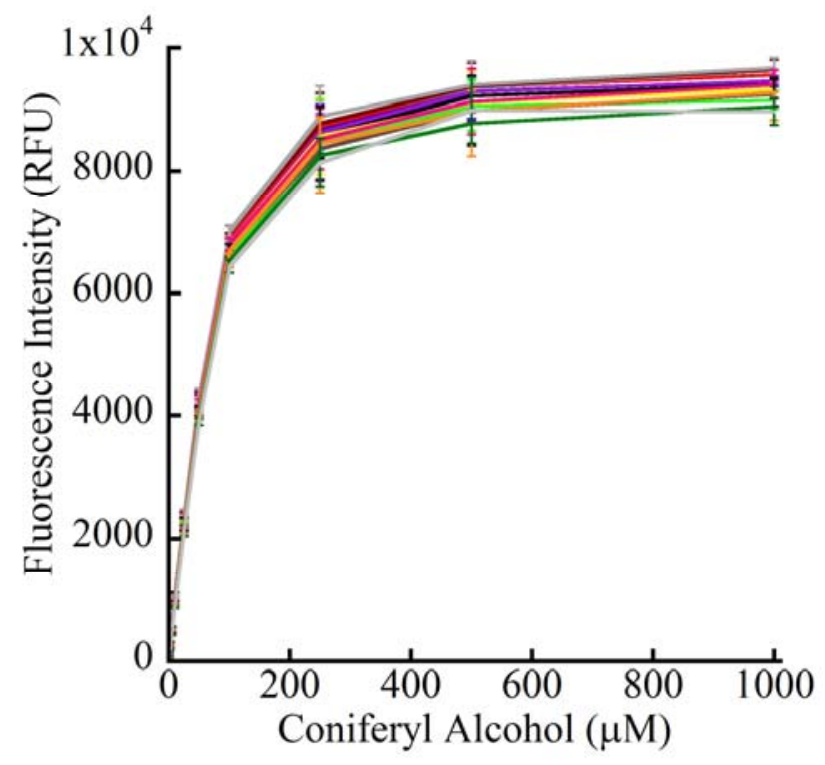

Figure 11B. Linearity of CA photostability. This figure shows the negligible effects on linearity between 1.0 to $100 \mu \mathrm{M}$ CA over the various interrogation times. The correlation of coefficient, $r^{2}$ was $0.985 \pm 0.001$ for $n=14$; an error of $<0.2 \%$ from CA samples subjected to 42 cycles of excitation/emission over a $24 \mathrm{~h}$ period. All other conditions and the colored tracings representing measurements between 5 and $1440 \mathrm{~min}$ are the same as those described for Figure 11A.

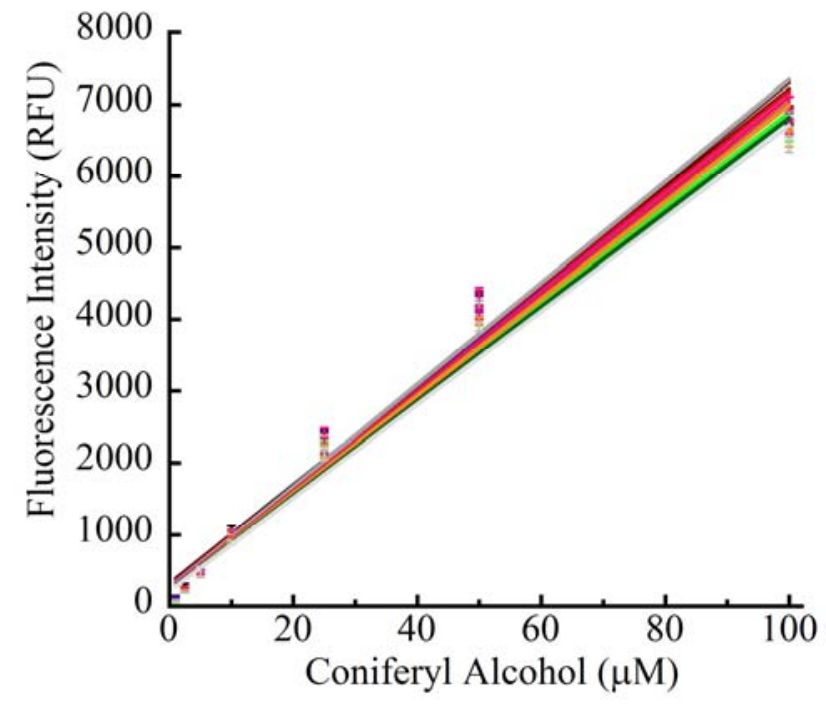


Photostability was also observed over 14 cycles of excitation and emission at each of the 3 different excitation wavelengths, resulting in a total of 42 cycles, further documenting photostability. The unwavering intensity profiles over time and several concentrations of CA are evident from the tracings being nearly parallel to the abscissa (Figure 12) and the overlapping tracings in Figures $11 \mathrm{~A}$ and $\mathrm{B}$. We conclude that the fluorescence properties of CA were stable at least over the duration of the HTS assays. Analyses in 384-well plate demonstrated the HTS capabilities and also corroborated the sensitivity data of Figures 10A and B.

Figure 12. Time dependence of CA photostability. This figure shows the photostability of CA over time. All other reaction conditions were as described for Figures 11A and B. The concentrations $(\mu \mathrm{M})$ of CA are as follows: 1.0 (open circles, black), 2.5 (closed circles, red), 5 (open squares, purple), 10 (closed squares, green), 25 (open triangles, yellow), 50 (closed triangles, aqua), 100 (open diamonds, maroon), 250 (closed diamonds, grey), 500 (crosses, magenta) and 1000 (plusses, blue).

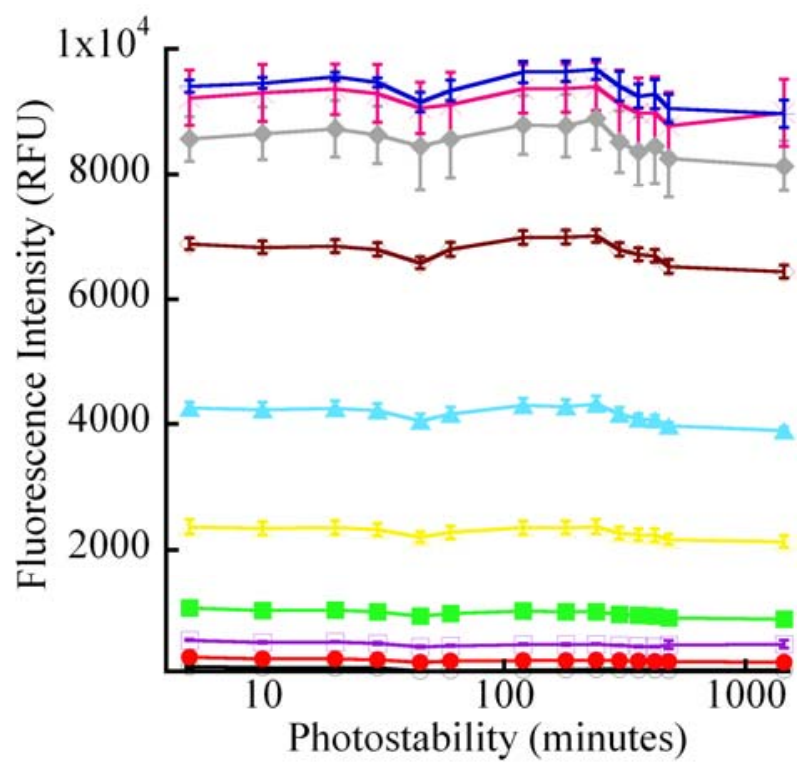

\section{Experimental}

\subsection{Materials}

Coniferyl alcohol (98\%-99\% pure according to the certificates of analysis) was purchased from Sigma-Aldrich (Saint Louis, MO), Alfa Aesar (Ward Hill, MA) and Acros Organics (Geel, Belgium) and used as received. According to Sigma-Aldrich's Fourier transform (FT) infrared (IR) Raman spectroscopy, FT-nuclear magnetic resonance (FT-NMR) spectroscopy and FT-IR spectroscopy data and Acros Organics NMR data posted on the company websites conformed to the structure. Stock solutions of CA, Trametes versicolor laccase (Sigma-Aldrich) and streptavidin-conjugated horseradish peroxidase (Pierce Chemical, Rockford, IL) were diluted in citrate buffer, $\mathrm{pH} 4.5$ (Fluka, purchased through Sigma-Aldrich) as described previously [6,8,9]. White 96- and 384-well microplates were from Perkin-Elmer (Waltham, MA). UV-transparent 96-well microplates were from Fisher Scientific (Pittsburgh, PA). Microplate-sealing tape was from Pierce. Citrate buffer, pH 4.5 was used for 
analyzing unmodified CA and laccase- or peroxidase-catalyzed CA oxidation. Basic $\mathrm{pH}$ reactions were done in $100 \mathrm{mM} \mathrm{NaOH}$ or $250 \mathrm{mM} \mathrm{KOH}$ (final pH after addition of base was $13.0 \pm 0.1$ ).

\subsection{Absorption Spectroscopy}

Absorption measurements were taken at $\sim 25^{\circ} \mathrm{C}$ as described previously [6,8]. The absorption of CA in $\mathrm{pH} 4.5$ buffer or in base was measured using 96-well UV-transparent microplate or quartz cuvette. Absorption spectra were generated by measuring the absorption intensities (A.U.) over the wavelengths $(\lambda)$ of 250 to $350 \mathrm{~nm}$ in $2 \mathrm{~nm}$ intervals using a microplate reader (Spectramax M2, Molecular Devices Sunnyvale, CA).

\subsection{Fluorescence Spectroscopy}

Fluorescence intensity (relative fluorescence units, RFU) from basified mixtures of CA and laccaseor peroxidase-oxidized CA were measured at $\sim 25{ }^{\circ} \mathrm{C}$ using 96- or 384-well microplate as described previously [8,9]. Samples were mixed by pipetting the solutions up and down using a multi-channel pipettor and/or by using the "automix" function of the microplate reader. The excitation spectrum was generated by holding the emission wavelength constant and varying the excitation over the wavelength of the absorption spectrum. Emission spectrum was generated with a fixed excitation wavelength. Fluorescence measurements were taken using Molecular Device M2 microplate reader.

\subsection{Enzymatic Oxidation}

Laccase- or peroxidase-catalyzed oxidation of CA was carried out at $\sim 25{ }^{\circ} \mathrm{C}$ in citrate buffer, $\mathrm{pH} 4.5$ using 96-well microplates [6,8,9]. Laccase oxidation was by mixing the enzyme and the substrate (CA) in buffer. Peroxidase oxidation involved mixing the enzyme and $\mathrm{CA}$ in buffer and initiating the catalysis by adding $\mathrm{H}_{2} \mathrm{O}_{2}\left(250 \mu \mathrm{M}\right.$ final). Alternately, CA and $\mathrm{H}_{2} \mathrm{O}_{2}$ were mixed and catalysis was initiated by adding the enzyme. Substrate, enzyme, buffer, base, peroxidase $+250 \mu \mathrm{M} \mathrm{H}_{2} \mathrm{O}_{2}$ without CA and $\mathrm{CA}+250 \mu \mathrm{M} \mathrm{H}_{2} \mathrm{O}_{2}$ without peroxidase were used as negative controls. Normally, kinetic reactions are monitored in real time. However, since basification was required in order to detect fluorescence, CA oxidation was monitored pseudo-kinetically by stopping identical enzymatic reactions at predetermined timed intervals using base or in endpoint mode after fixed time by basification [6]. During pseudo-kinetics, reaction wells were mixed intermittently using the "automix" function. The CA fluorescence and absorption decreased upon oxidation. The magnitude of decrease was a measure of catalysis [6].

\subsection{Halide Inhibition}

Sodium chloride was added to 96-well white microplate at the start of $0.5 \mu \mathrm{g} / \mathrm{mL}$ laccase oxidation of $250 \mu \mathrm{M} \mathrm{CA}$ at $\sim 25{ }^{\circ} \mathrm{C}$ for 60 min or after terminating the reactions with base. The former set of samples demonstrated the inhibitory effects of halide ions on laccase oxidation of CA. The latter set showed the effects (if any) of ionic strength on the fluorescence of oxidized CA. As an additional control, the effects of ionic strength on the fluorescence of unmodified CA ( $250 \mu \mathrm{M})$ were also tested. Additional details are in [8]. 


\subsection{Stability Study}

Ten different concentrations of CA, spanning three orders of magnitude ( 1 to $1000 \mu \mathrm{M}$ ) were prepared in $100 \mathrm{mM} \mathrm{NaOH}$ and added in 25 or $50 \mu \mathrm{L}$ volumes to 384-well white microplate. CA samples were interrogated over 24 hours at 14 timed intervals (5 to $1440 \mathrm{~min}$ ) using three different excitation wavelengths (290, 310 and $340 \mathrm{~nm}$ ). Emission was monitored at $416 \mathrm{~nm}$. To minimize the evaporation, microplate sealing tape was used between measurements. To protect the samples from light, the plate was also covered with an aluminum foil between measurements.

\subsection{Data Analysis}

All reactions were carried out at least in triplicate. Results were calculated as average \pm standard deviation. Linearity (correlation of coefficient, $r^{2}$ ), limits of detection (LOD) and quantitation (LOQ) were calculated as described previously $[6,8,9]$. The magnitude of fluorescence decrease following oxidation was calculated by subtracting the RFU values of enzymatic reactions (signal, $S$ ) from the RFU values of CA (blank, B) (i.e., B - S) and was designated as delta RFU ( $\triangle$ RFU). Since the magnitude of fluorescence decrease increased with time or enzyme concentration, the $\Delta$ RFUs assumed a positive character. The S/B was also used to evaluate certain data.

\section{Conclusions}

The origin of lignin fluorescence is not clear due to structural complexities, role of solvent and other factors [15]. Lignin was reported to fluoresce at 360-395 nm after excitation over 240-340 nm wavelengths [16,17], whereas lignin model compounds fluoresced at 370-465 nm [18]. Environmental sampling for lignin involved excitation over the wavelengths of $250-440 \mathrm{~nm}$ and monitoring the emission between 300-600 nm [19]. It is important to note that we have not tested environmental samples for interference in our fluorescent assays of CA. Lignin-CA polymer and cell wall-bound ferulic acid were identified as major fluorophores over the 440-530 nm emission wavelengths [20-22]. Ferulic acid and CA are identical except for the carboxyl group in the former replacing the hydroxyl group of the latter. We believe that this report is the first comprehensive description of the fluorescence properties of CA and fluorescence diminishment consequent to laccase and peroxidase oxidation. We described the advantages of the CA absorption assays recently [6]. These advantages are also relevant to the CA fluorescent assays and can be used in the coupled assays for enzymes that utilize, generate or liberate CA. These include vanillyl alcohol oxidase or eugenol oxidase that convert eugenol to CA and $\beta$-glucosidase that releases CA from coniferin [6, and references cited therein]. We hope to eventually deploy an orthogonal, field-portable, multiplexed, HTS biosensor for the in situ, onsite, standoff interrogation of plant biomass monolignols using absorption [6], fluorescence [8,9] and electrochemical [9] techniques. Our studies might aid in the rational selection of pretreatment strategies based on lignin compositional analyses in order to improve saccharification through the reduction of biomass recalcitrance for the production of cost-effective biofuels [1-3]. 


\section{Acknowledgements}

The DOE's JBEI (http://www.jbei.org) is supported by the U.S. Department of Energy, Office of Science, Office of Biological and Environmental Research, through Contract DE-AC02-05CH11231 between Lawrence Berkeley National Laboratory and the U.S. Department of Energy. Sandia is a multiprogram laboratory operated by Sandia Corp., a Lockheed Martin Company, for the United States Department of Energy under Contract DE-AC04-94AL85000.

\section{References and Notes}

1. Boerjan, W.; Ralph, J.; Baucher, M. Lignin biosynthesis. Annu. Rev. Plant Biol. 2003, 54, 519-546.

2. Weng, J.K.; Li, X.; Bonawitz, N.D.; Chapple, C. Emerging strategies of lignin engineering and degradation for cellulosic biofuel production. Curr. Opin. Biotechnol. 2008, 19, 166-172.

3. Simmons, B.A.; Loque, D.; Blanch, H.W. Next-generation biomass feedstocks for biofuel production. Genome Biol. 2008, 9, 242.1-242.6.

4. Leonowicz, A.; Cho, N.S.; Luterek, J.; Wilkolazka, A.; Wojtas-Wasilewska, M.; Matuszewska, A.; Hofrichter, M.; Wesenberg, D.; Rogalski, J. Fungal laccase: properties and activity on lignin. J. Basic Microbiol. 2001, 41, 185-227.

5. Ruiz-Duenas, F.J.; Martinez, A.T. Microbial degradation of lignin: how a bulky recalcitrant polymer is efficiently recycled in nature and how we can take advantage of this. Microbial. Biotechnol. 2009, 2, 164-177.

6. Achyuthan, K.E.; Adams, P.D.; Simmons, B.A.; Singh, A.K. Spectroscopic analyses of the biofuels-critical phytochemical, coniferyl alcohol and its enzyme-catalyzed oxidation products. Molecules 2009, 14, 4758-4778.

7. Achyuthan, K.E.; Whitten, D.G. Design considerations for high-throughput screening and in vitro diagnostic assays. Comb. Chem. High Throughput Screen. 2007, 10, 399-412.

8. Achyuthan, K.E.; McClain, J.L.; Raj, D. Orthogonal spectroscopic high-throughput screening of laccase-catalyzed p-cresol oxidation. Comb. Chem. High Throughput Screen. 2009, 12, 678-689.

9. Harper, J.C.; Brozik, S.M.; Flemming, J.H.; McClain, J.L.; Polsky, R.; Raj, D.; Ten Eyck, G.A.; Wheeler, D.R.; Achyuthan, K.E. Fabrication and testing of a microneedles sensor array for $p$ cresol detection with potential biofuel applications. ACS Appl. Mater. Interfaces 2009, 1, 1591-1598.

10. Zuck, P.; O’Donnell, G.T.; Cassaday, J.; Chase, P.; Hodder, P.; Strulovici, B.; Ferrer, M. Miniaturization of absorbance assays using the fluorescent properties of white microplates. Anal. Biochem. 2005, 342, 254-259.

11. Redmond, R.W. Introduction to fluorescence and photophysics. In Handbook of Biomedical Fluorescence; Mycek, M.A., Pogue, B.W., Eds.; Marcel Dekker Inc.: NewYork, NY, USA, 2003; pp. 1-28.

12. Demchenko, A.P. Optimization of fluorescence response in the design of molecular biosensors. Anal. Biochem. 2005, 343, 1-22.

13. Segel, I.H. Enzyme Kinetics; Wiley-Interscience: NewYork, NY, USA, 1975; p. 957. 
14. $\mathrm{Xu}, \mathrm{F}$. Effects of redox potential and hydroxide inhibition on the $\mathrm{pH}$ activity profile of fungal laccases. J. Biol. Chem. 1997, 272, 924-928.

15. Olmstead, J.A.; Gray, D.G. Fluorescence spectroscopy of cellulose, lignin and mechanical pulps: a review. J. Pulp Paper Sci. 1997, 23, J571-J581.

16. Radotic, K.; Kalauzi, A.; Djikanovic, D.; Jeremic, M.; Leblanc, R.M.; Cerovic, Z.G. Component analysis of the fluorescence spectra of a lignin model compound. J. Photochem. Photobiol. B: Biol. 2006, 83, 1-10.

17. Lundquist, K.; Josefsson, B.; Nyquist, G. Analysis of lignin products by fluorescence spectroscopy. Holzforschung 1978, 32, 27-32.

18. Albinsson, B.; Li, S.; Lundquist, K.; Stomberg, R. The origin of lignin fluorescence. J. Mol. Struct. 1999, 508, 19-27.

19. Hernes, P.J.; Bergamaschi, B.A.; Eckard, R.S.; Spencer, R.G.M. Fluorescence-based proxies for lignin in freshwater dissolved organic matter. J. Geophys. Res. 2009, 114, G00F03.

20. Djikanovic, D.; Kalauzi, A.; Jeremic, M.; Micic, M.; Radotic, K. Deconvolution of fluorescence spectra: contribution to the structural analysis of complex molecules. Colloids Surf. B. Biointerfaces 2007, 54, 188-192.

21. Lichtenthaler, H.K.; Schweiger, J. Cell wall bound ferulic acid, the major substance of the bluegreen fluorescence emission of plants. J. Plant Physiol. 1998, 152, 272-282.

22. Meyer, S.; Cartelat, A.; Moya, I.; Cerovic, Z.G. UV-induced blue-green and far-red fluorescence along wheat leaves: a potential signature of leaf aging. J. Exp. Bot. 2003, 54, 757-769.

Sample Availability: Samples of the compounds are available from the authors.

(C) 2010 by the authors; licensee Molecular Diversity Preservation International, Basel, Switzerland. This article is an open-access article distributed under the terms and conditions of the Creative Commons Attribution license (http://creativecommons.org/licenses/by/3.0/). 